\title{
MRI Relaxometry: Methods and Applications
}

\author{
A. A. O. Carneiro, G. R. Vilela, D. B. de Araujo, and O. Baffa \\ Departamento de Física e Matemática, FFCLRP, Universidade de São Paulo, 14040-901, Ribeirão Preto - SP, Brazil
}

Received on 21 October, 2005

\begin{abstract}
Aspects of magnetic resonance relaxation measurements in human tissues are discussed. The influence of pulse sequences and parameters are compared and analyzed for different tissues. By controlling the acquisition parameters and data fitting the relaxation rate can be useful in several clinical situations. The influence of repetition and echo time, predicted in sequences of signal acquisition, on measurement of transversal relaxation time (T2) was evaluate using simulated MRI signal.
\end{abstract}

Keywords: Magnetic resonance imaging; MRI

\section{INTRODUCTION}

The use of magnetic resonance imaging (MRI) as a quantitative tool has attracted great interest by various research centers. The improvement in the sensitivity and the reduction of the subjectivity of visual evaluation created a significant impact on diagnosis of tissue abnormalities, such as tissue iron overload.

The most common MRI techniques for quantitative diagnosis at the lesion level are relaxometry (R), magnetization transfer (MT) and Spectroscopy (MRS). However, one important issue is standardizing a calibrating protocol to be used in different scanners that is imperative to allow the use of MRI as a quantitative tool.

Most commonly, MRI can be weighted in T2 (transversal relaxation time) and/or T1 (longitudinal relaxation time), where the nature of the image contrast is based on relative contributions from different tissues. On the other hand, one can create a map, based on the relaxation time itself, also known as relaxometry. Such map can be constructed considering each one of these parameters separately or together. Generally, applications for tissue characterization make use of spin-echo sequences, with two or more different times of echo (TE) and a long time of repetition (TR), and are currently being applied to evaluate brain, breast, muscle and liver tissue [1-7].

We present here the relevant principles of T2 weighted relaxometry. Section 2 reviews important aspects of relaxation rate (R2) for specific tissues and specific MRI sequences dedicated to relaxometry. With these sequences an R2 map can be produced and overlaid onto the images. Section 2.1 discusses the condition under which the TE for each tissue optimizes the sensitivity of the exam. In section 2.2 we present image acquisition and processing that allows the best fit between signal intensity and TE. Details and results of particular applications to evaluate tissue lesions and liver iron overload by relaxometry are discussed on section 3 . The variance between the experimental data and fitted curve will also be discussed.

\section{THEORY AND METHODS}

The theory of relaxometry, or the measurement of relaxation rates, is based on the physical aspects of nuclei relax- ation to the ground state after being excited by an RF pulse. This relaxation is produced by randomly occurring variations in the local magnetic field strength. T2 relaxation is due to a dephasing of individual magnetic moments of the protons, and begins immediately after the RF pulse. Protons will become out of phase as they experience a slightly different magnetic field and then rotate at a slightly different frequency. This transversal relaxation occurs both due to magnetic field inhomogeneities produced by the magnet or by magnetic particles present in the tissues and due to movement of the molecules in the tissue.

\section{A. Imaging acquisition and processing}

Relaxometry maps may be generated either by spin-echo or by gradient echo sequences. In the latter, $\mathrm{T} 2 *$ is being measured, rather than T2. Therefore, the results may be noisier since the influence of inhomogeneities in the magnetizing field is greater.

To generate a map of relaxation rate $(\mathrm{R} 2=1 / \mathrm{T} 2)$ or relaxation time (T1 or T2), using spin echo sequences, at least two images are necessary. The sensitivity of this technique depends on sequence, time of repetition (TR), time of echo (TE), the number of images acquired with different TE, and on the model adopted for fitting the experimental data.

When using a single spin echo acquisition, for T2 weighted images, the selected TEs values should be close of the T2 of the tissue. If TE is much longer than $\mathrm{T} 2$, the fit will be weighted by the relaxed part of signal intensity versus TE. In general, relaxometry uses a multi-spin-echo sequence (MSE). In this case, if the first echo was much shorter than $\mathrm{T} 2$ of the tissue, a larger number of echoes will be necessary to evaluate a high value of $\mathrm{T} 2$. This means a high repetition rate and, consequently, thermal effects will contribute to dephase the spins, making T2 even shorter. On the other hand, if the first TE is long the delay between two images will allow the acquisition of only a few images in the range of TE to best calculate T2. One possibility to avoid this incompatibility of an MSE is to use single spin-echo sequence (SSE), and repeat the acquisition for different echoes. Unfortunately, this method will need an undesirably long time of scanner [8]. In the case of very short T2 short values of TE will be necessary. St Pierre et al. 
showed that a single spin echo sequence may be used to improve the precision of the relaxometry technique to evaluate iron overload in tissue [9].

Increasing TR also increases the signal-to-noise ratio (SNR) in relaxometry evaluation. Generally, TR is at least three times T1. However, increasing TR increases the acquisition time. Another important aspect in relaxometry is the value and the number of TEs. The larger the number of TEs the better is the SNR .....[9]. The values of TE should be chosen in a range centered close to the values of $\mathrm{T} 2$ for the sample. A value of T2 close to TE, but sufficiently longer than T2 fluctuations in region of interest (ROI) of the tissue, will give more accurate $\mathrm{T} 2$ measurements.

For tissues where the signal intensity decays rapidly, such as tissues with iron overload, the signal intensity (SI) versus TE is well fit by a single-exponential function [7,10]. Moreover, SNR increases when the images are pre-processed by a smooth filter. However, this process decreases the spatial resolution of the relaxometry map [11]. It is common to quantify $\mathrm{T} 2$ values by the regression of the natural logarithm of the signal intensity versus TE with two exponential functions. The T2 determination is uncertain if the relaxation of the SI does not follow a single-exponential behavior. An even more serious uncertainty in T2 results appears if an offset signal is not subtracted from the SI before the curve fitting. In practice, a single exponential decay of the signal intensity (SI) versus TE should not be applied for all tissues as it will be show in the next section.

In quantitative magnetic resonance imaging, the accuracy of an image derived from relaxation rates is primarily dependent on the processing of the image data that must be uncorrupted by acquisition artifacts [12]. Respiratory motion, for example, is a predominant source of artifacts, especially for axial MR images of the abdomen [13]. Besides traditional noise suppression, another important method of image processing is providing a reference signal intensity to compensate differences of image brightness from the same tissue acquired with different TE. One can use a homogeneous phantom with an MRI signal intensity equivalent to the average signal of the tissue being investigated. Therefore, the value of the intensity from the phantom for each TE may be used to correct the scanner drift of the signal intensities from the tissue being investigated [6].

\section{B. T2 analysis by simulated data}

To the first approximation, the relaxation of signal intensity, following a spin-echo pulse sequence assumes an exponential decay, given by the expression [14]:

$$
S_{S E}=S_{o}\left[1-2 e^{-\frac{T R-T E / 2}{T_{1}}}+e^{-\frac{T E}{T_{1}}}\right] e^{-\frac{T E}{T_{2}}},
$$

where $\mathrm{S}_{0}$ is the proton density, $\mathrm{T} 1$ and $\mathrm{T} 2$ are longitudinal and transversal relaxations, respectively. The parameters $\left(\mathrm{S}_{o}\right.$, $\mathrm{T}_{1}$ and $\mathrm{T}_{2}$ ) characterize the tissue properties and the image contrast should be weighted with each one of these parameters by controlling TE and TR during the image acquisition.

In practice, the use of equation 1 to fit the $S_{S E}$ versus TE curve poses a difficult task. Equation 1 does not separate T1 and $\mathrm{T} 2$ contributions sufficiently to detect fine abnormalities of tissues. Generally, one can eliminate T1 contribution from expression 1 by using a TR much longer than T1, as most biological tissues have a T1 shorter than $250 \mathrm{~ms}$. When the tissue has a long T1, as in the Cerebral Spinal Fluid (CSF) (T1 2500 ms and T2 1400 ms), a very long TR will be necessary in order for T2 to dominate the signal decay.

The dependency of the MRI signal on TE was simulated using equation 1 for different values of T1, T2 and TR. As shown in Fig. 1, the signals were plotted using. The follow procedure: a) large variation on $\mathrm{T} 1(500,1000,1500)$, $\mathrm{T} 2=100 \mathrm{~ms} ; \mathrm{TR}=2500$ and $\mathrm{S}_{0}=1000 ;$ b) $10 \%$ of variation on $\mathrm{T} 2(90,100,110), \mathrm{T} 1=500 \mathrm{~ms}, \mathrm{TR}=5 \times \mathrm{T} 1$ and $\mathrm{S}_{0}=$ 1000 ; c) $10 \%$ of variation on $\mathrm{S}_{0}(900,1000,1100), \mathrm{T} 2=$ $100 \mathrm{~ms}, \mathrm{~T} 1=500, \mathrm{TR}=5 \times \mathrm{T} 1 \mathrm{~ms}$; d) large variation on $\mathrm{TR}$ $(1000,2000,3000), \mathrm{T} 2=100 \mathrm{~ms}, \mathrm{~T} 1=1000 \mathrm{~ms}$ and $\left.\mathrm{S}_{0}=1000\right)$. A Gaussian noise of $1 \%$ was added to all simulated signals to represent statistical fluctuations of detection system and the random variations of the local electromagnetic field due to eddy currents induced within tissues.

The simulated signals were fitted using single and biexponential curve, according to:

$$
\begin{gathered}
S_{S E}=S_{o} e^{-\frac{T E}{T_{2}}}+S_{\text {offset }}, \quad(\text { single }-) \\
S_{S E}=S_{o 1} e^{-\frac{T E}{T 2_{1}}}+S_{o 2} e^{-\frac{T E}{T 2_{2}}}+S_{\text {offset }}, \quad(\mathrm{bi}-)
\end{gathered}
$$

where $\mathrm{S}_{o}$ is the maximum signal from the sample and $\mathrm{S}_{\text {offset }}$ is an offset signal from the system.

To evaluate the variation on parameters $S_{0}$ and $T 2$, the fit was made considering two methods: all arbitrary parameters free and some parameters fixed. This second one was made using the following protocol:

Single-exponential: offset was considered fixed and equal to zero;

Bi-exponential: $S_{01}$ equal to $S_{02}$ and the offset was fixed equal to the noise level estimated for each signal. In the simulated data the offset was zero.

Simulations were performed using a program written in MatLab 6.5 and the relaxometry parameters were estimated by fitting the simulated data using a non-linear curve fit according to $\chi^{2}$ minimization algorithm, based on LevenbergMarquardt method, with an interval of confidence of $95 \%$.

The relaxation curves simulated for three different $\mathrm{T} 2$ (Fig. 1.A) show a maximum variance in the region where TE is approximately equal to $\mathrm{T} 2$. $10 \%$ of variance on proton density (Fig. 1.B) implies one visible variance on the decay of the signal intensity. It was observed that a large variance on $\mathrm{T} 1$ causes small variance on signal intensity for $\mathrm{TR}=5 \mathrm{~T} 1$. Large variations on T1 of the tissue won't cause considerable modification on relaxometry, if TR $>>\mathrm{T} 1, \mathrm{~T} 2$. However, if TR is 

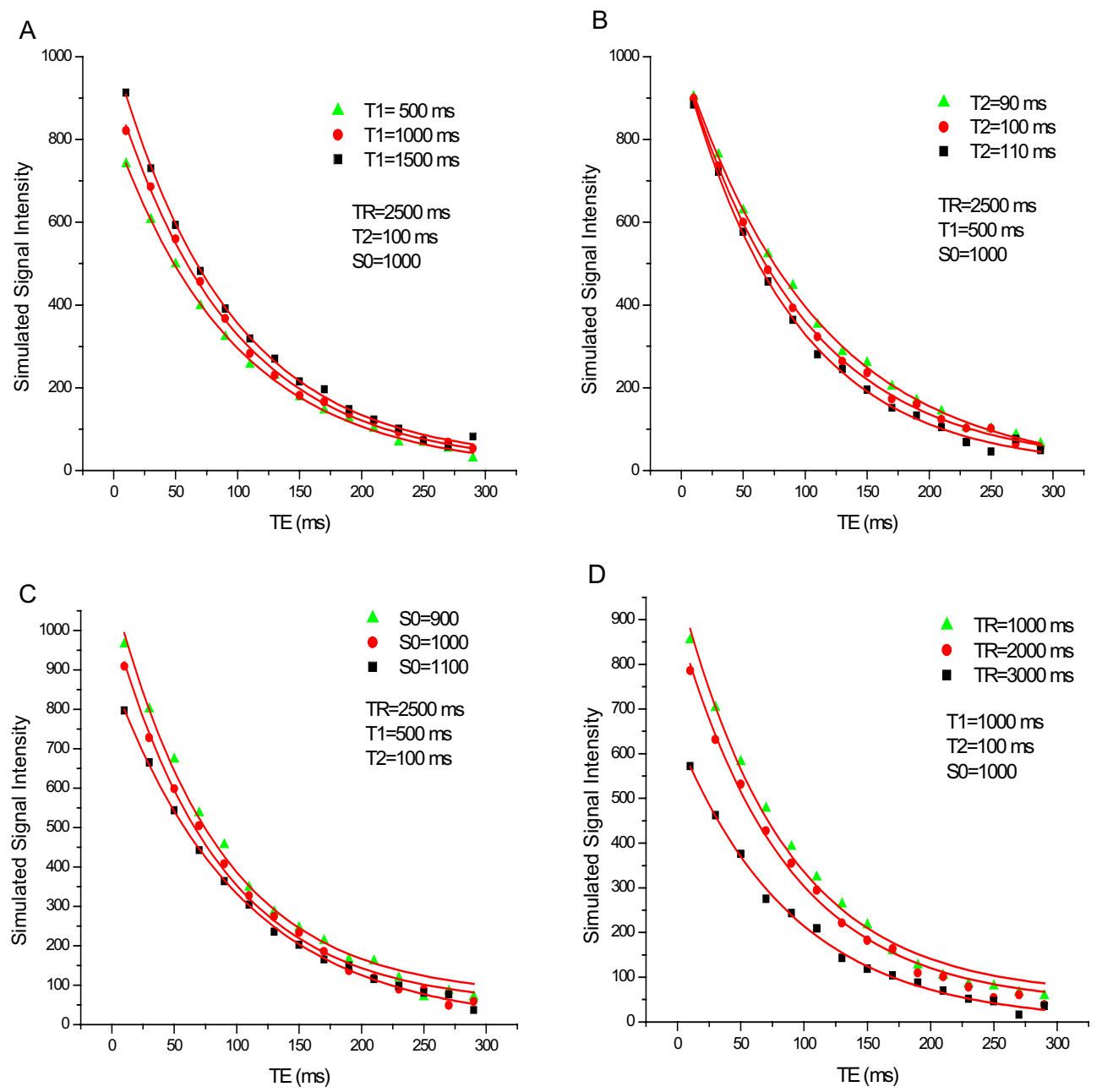

FIG. 1: Signal intensity versus TE values simulated from equation 1 and fitted by equation 2 . a) large variation on T1, with TR $>>$ T1; b) 10\% of variation on $\mathrm{T} 1$ for $\mathrm{TR}=2.5 * \mathrm{~T} 1$; c) $10 \%$ of variation on $\mathrm{T} 2$, with $\mathrm{TR}=2,500 \mathrm{~ms}, \mathrm{~T} 1=500 \mathrm{~ms}$; $) \mathrm{TR}=[\mathrm{T} 1,2 \mathrm{~T} 1 \mathrm{and} 3 \mathrm{~T} 1]$, with $\mathrm{T} 1=1,000 \mathrm{~ms}$, $\mathrm{T} 2=100 \mathrm{~ms}$.

equivalent to T1, small variations will result in a considerable modification on signal amplitude.

Table 1 and 2 show the distribution of relaxometry parameters obtained from single- and bi-exponential fit of the simulated curves of Fig. 1. Although the offset level $\left(\mathrm{S}_{\text {offset }}\right)$ has been considered zero in the simulated data, a fluctuation of about $1 \%$, relative to proton density $\left(\mathrm{S}_{0}\right)$ was observed. The shorter TR, the larger is the deviation of T2 obtained both by single- and by bi-exponential fit.

The absolute deviation of $\mathrm{T} 2$ for all conditions of simulation presented in table 1 and 2 was smaller when T2 was obtained considering the offset fixed and equal zero and with equal amplitudes of $S_{01}$ and $S_{02}$ for the bi-exponential curve. For these parameters, the value obtained for $\mathrm{T} 2$ was similar for both single and bi-exponential fits.

When all parameters of equation 3 were allowed to be adjusted, the fluctuation in both $\mathrm{T} 22_{1}$ and $\mathrm{T} 22_{2}$ was large. Fig. 3 shows that using a bi-exponential fit (equation 3), results in large uncertainties in T2 values. Besides, the number of iterations necessary to have the fitted data converging to a $95 \%$ confidence is greater than when some parameter is maintained fixed.

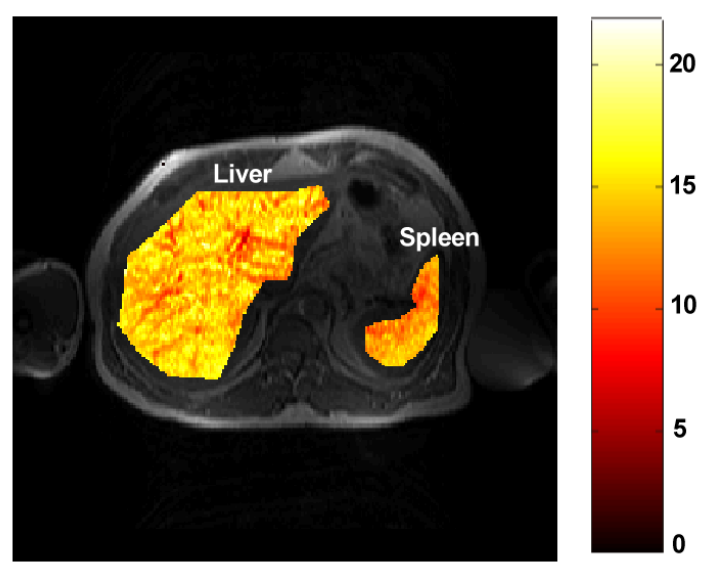

FIG. 2: Map of relaxation rate R2 (1/T2) on Liver and spleen region superimposed on anatomic MRI image acquired on 40 year old man. 
TABLE I: Relaxometry parameter obtained from Single- and Bi-exponential fitting of simulated MRI signal from a spin-echo sequence versus TE. In this table $\bar{T} 2$ is the average value of T2. Shadowed cells show the varying parameters.

\begin{tabular}{|c|c|c|c|c|c|c|c|c|c|c|c|c|}
\hline \multicolumn{4}{|c|}{ Simulated parameters } & \multicolumn{3}{|c|}{$\begin{array}{l}\text { Single-exponential } \\
S=S_{0} e^{-\frac{T E}{T 2}}+S_{\text {offset }}\end{array}$} & \multicolumn{6}{|c|}{$\begin{array}{l}\text { Bi-exponential } \\
S=S_{01} e^{-\frac{T E}{T 2_{1}}}+S_{02} e^{-\frac{T E}{T 2_{2}}}+S_{\text {offset }}\end{array}$} \\
\hline S0 & TR & $\mathrm{T} 1$ & $\mathrm{~T} 2$ & $\mathrm{~S}_{\text {offset }}$ & $\mathrm{A}$ & $\mathrm{T} 2$ & $\mathbf{S}_{\text {offset }}$ & $\mathrm{S}_{01}$ & $\mathrm{~S}_{02}$ & $\mathrm{~T} 2,1$ & $\mathrm{~T} 2,2$ & $\bar{T} 2$ \\
\hline 1000 & 2500 & 500 & 90 & 7.2 & 988.5 & 89.1 & 7.2 & 491.2 & 497.3 & 89.0 & 89.0 & 89.0 \\
\hline 1000 & 2500 & 500 & 100 & 13.4 & 982.7 & 96.1 & 2.5 & 486.0 & 495.0 & \begin{tabular}{|l|}
96.9 \\
\end{tabular} & 103.9 & 100.4 \\
\hline 1000 & 2500 & 500 & 110 & -5.0 & 1003 & 109.3 & 2.9 & 496.0 & 499.0 & 99.9 & 114.0 & 107.0 \\
\hline 1000 & 2500 & 1000 & 100 & 11.4 & 914.3 & 94.2 & 6.1 & 444.3 & 468.0 & 102.8 & 90.7 & 96.8 \\
\hline 1000 & 2500 & 1500 & 100 & -4.1 & 824.0 & 99.3 & 7.2 & 385.8 & 428.8 & 101.5 & 91.4 & 96.5 \\
\hline 1000 & 2000 & 500 & 100 & -12.0 & 878.9 & 101.7 & 15.9 & 417.2 & 449.5 & 95.2 & 90.6 & 92.9 \\
\hline 1000 & 3000 & 500 & 100 & -10.0 & 957.2 & 102.7 & 15.2 & 435.8 & 501.0 & 98.8 & 94.5 & 96.7 \\
\hline 900 & 2500 & 500 & \begin{tabular}{|l|}
100 \\
\end{tabular} & 4.2 & 879.8 & 101.1 & 6.9 & 403.0 & 477.9 & 105.6 & 95.3 & 100.5 \\
\hline 1100 & 2500 & 500 & 100 & 4.8 & 1067 & 101.4 & 8.4 & 489.7 & 578.8 & 105.6 & 95.5 & 100.6 \\
\hline
\end{tabular}

TABLE II: Relaxometry parameter obtained from Single- and Bi-exponential fitting of simulated MRI signal from a spin-echo sequence versus TE. In this fit, the offset was maintained fixed. Shadowed cells show the varying parameter.

\begin{tabular}{|l|l|l|l|l|l|l|l|l|l|l|l|}
\hline \multicolumn{3}{|l|}{ Simulated parameters } & \multicolumn{3}{l}{$\begin{array}{l}\text { Single-exponential } \\
S=S_{0} e^{-\frac{T E}{T 2_{1}}}\end{array}$} & \multicolumn{3}{l}{ Bi- exponential $S=S_{0}\left(e^{-\frac{T E}{T 2_{1}}}+e^{-\frac{T E}{T 2_{2}}}\right)$} \\
\hline $\mathrm{S}_{0}$ & TR & $\mathrm{T} 1$ & $\mathrm{~T} 2$ & $\mathrm{~S}_{\text {offset }}$ & $\mathrm{S}_{0}$ & $\mathrm{~T} 2$ & $\mathrm{~S}_{0 \text { ffset }}$ & $\mathrm{S}_{0}$ & $\mathrm{~T} 2_{1}$ & $\mathrm{~T} 2_{2}$ & $\bar{T} 2$ \\
\hline 1000 & 2500 & 500 & 90 & 0.0 & 991.0 & 91.2 & 0.0 & 495.0 & 91.7 & 91.7 & 91.7 \\
\hline 1000 & 2500 & 500 & 100 & 0.0 & 988.3 & 100.2 & 0.0 & 494.1 & 100.2 & 100.2 & 100.2 \\
\hline 1000 & 2500 & 500 & 110 & 0.0 & 1000 & 107.7 & 0.0 & 510.0 & 107.6 & 107.6 & 107.6 \\
\hline 1000 & 2500 & 1000 & 100 & 0.0 & 918.9 & 97.8 & 0.0 & 459.4 & 97.2 & 97.2 & 97.2 \\
\hline 1000 & 2500 & 1500 & 100 & 0.0 & 822.2 & 97.8 & 0.0 & 411.1 & 97.8 & 97.8 & 97.8 \\
\hline 1000 & 2000 & 500 & 100 & 0.0 & 873.7 & 97.3 & 0.0 & 436.0 & 97.3 & 97.3 & 97.3 \\
\hline 1000 & 3000 & 500 & 100 & 0.0 & 952.5 & 99.6 & 0.0 & 476.5 & 99.6 & 99.6 & 99.6 \\
\hline 900 & 2500 & 500 & 100 & 0.0 & 881.7 & 102.6 & 0.0 & 490.8 & 102.6 & 102.6 & 102.6 \\
\hline 1100 & 2500 & 500 & 100 & 0.0 & 1070 & 102.6 & 0.0 & 534.6 & 102.7 & 102.7 & 102.7 \\
\hline
\end{tabular}

\section{SINGLE- VERSUS BI-EXPONENTIAL FIT}

Generally, T2 has been evaluated using single- or biexponential fitting to the experimental data with no attention given to the mathematical protocol to best adjust appropriate parameters. For example, the value of T2 obtained from an exponential fit could be strongly influenced by the choice of the amplitude of the signal offset $\left(\mathrm{S}_{\text {offset }}\right)$. A bi-exponential function makes the fitting more critical, since besides the variation of amplitude of offset, the exponential terms will interact to permit the best fit. In this case, both the amplitude and relaxation time parameters fluctuate between mean values. This criticality is clearly observed from simulated results presented in section 2.1. For example, for 5 simulated signals with $1 \%$ of noise, generated using the same parameters $(\mathrm{TR}=2,500 \mathrm{~ms}$, $\mathrm{T} 1=500 \mathrm{~ms}$, and $\mathrm{T} 2=100 \mathrm{~ms}$ ) the fluctuation of the adjusted parameter was about $0.8 \%$ when the single-exponential fitting was done keeping the offset fixed and about $4 \%$ when all other parameters were free. The results in table 1 and 2 show the criticality of the bi-exponential fit when all 5 parameters were free to be adjusted. The adjusted parameters were more stable when both amplitudes were considered equal and the offset was fixed. This mathematical procedure of getting the relaxometry parameters guarantees the best resolution of this procedure to detect small relaxation changes of tissues.

\section{IN VIVO APPLICATIONS}

In this section we will present examples of relaxometry applications to quantify $\mathrm{T} 2$ or R2 values in some biological tissues. To guarantee a relative comparison between different tissues, the same sequence and processing were used to generate R2 maps. Images were acquired in a $1.5 \mathrm{~T}$ scanner (Siemens Magneton Vision) using a multi-spin-echo sequence (16 echoes) with TE multiples of $22.5 \mathrm{~ms}[22.5,45, \ldots 360$ $\mathrm{ms}$, with long $\mathrm{TR}\left(\mathrm{TR}_{i}=2,000 \mathrm{~ms}\right)$. Due to the long repetition time, no respiratory-gating or breath-holding techniques were employed to avoid movement artifacts. R2 maps were superposed onto an intensity image for best visualization.

\section{A. Tissue Iron Overload}

The higher the concentration of iron stored on the tissue, the shorter will be the value of T2 (longer R2), and the shorter should be the TE values. Generally, patients that regularly receive blood transfusions have the level of iron in the body increased, especially those who are not submitted to iron chelation therapy. The organs that most accumulate iron are liver, heart and spleen.

Relaxometry is a potential tool to quantify iron overload in tissue $[5-8,14]$. But its accuracy depends strongly on good 
A

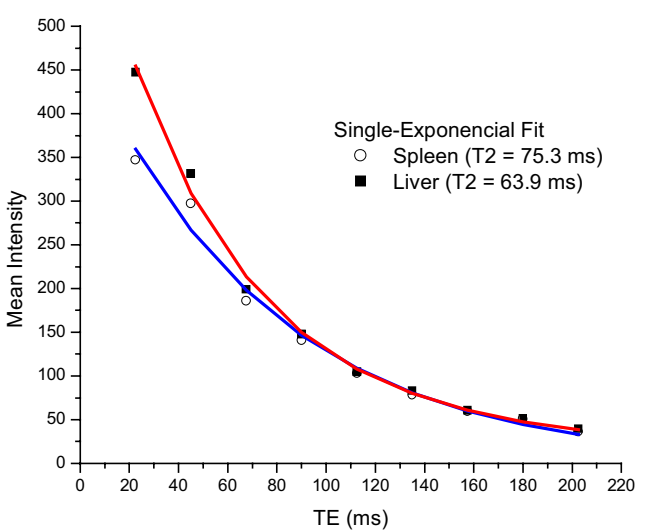

B

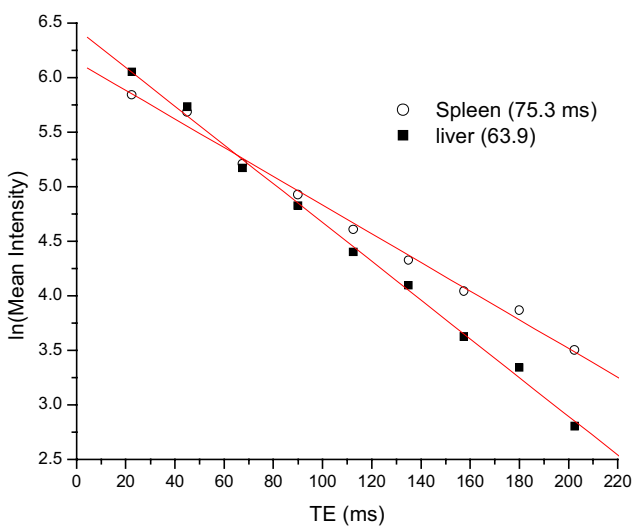

FIG. 3: Single-exponential fit of mean intensity signal versus TE for liver and spleen region drawn on Fig. 2 shown in a linear (A) and logarithmic (B) scale.

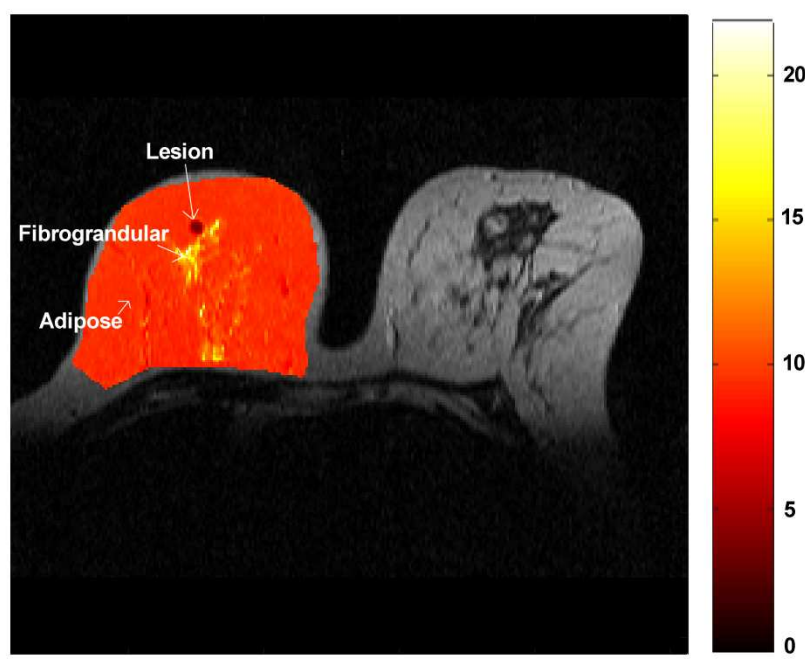

FIG. 4: Map of relaxation rate R2 (1/T2) of the left breast superposed on T2-weighted image. This image was acquired on a 54 year old woman.

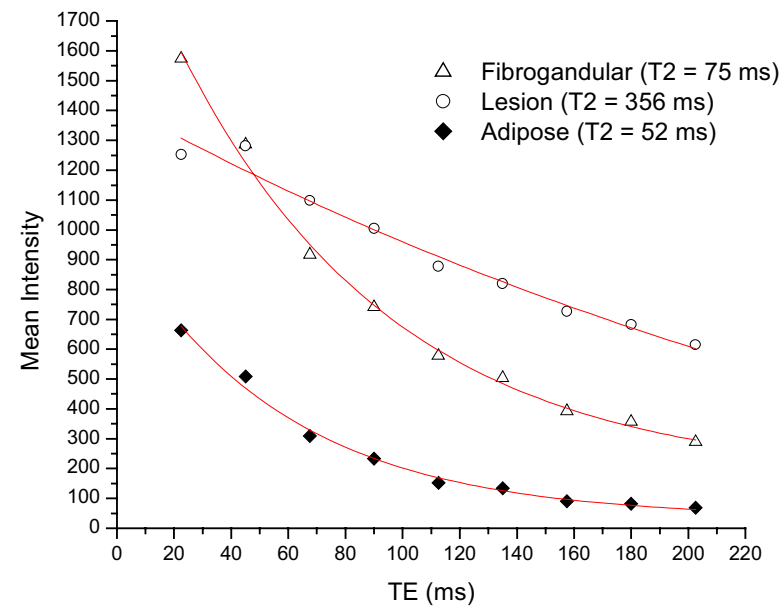

FIG. 5: Single-exponential fit of mean intensity signal versus TE from three different region of the breast: fibrogandular tissue $(\Delta)$; damaged tissue $(o)$ and adipose tissue $(\diamond)$ of the whole left breast area.

\section{B. Breast relaxometry}

controls, on the protocol of measurement and on processing [9].

Figure 2 shows an R2 map, acquired in an asymptomatic subject (40 year old), from the liver and spleen region. The R2 was obtained by fitting the signal intensity in a pixel-by-pixel basis using a single-exponential curve. A $3 \times 3$ matrix mask smooth filter was applied to reduce respiratory artifacts. As liver and spleen tissues have a short T2 $(\sim 40 \mathrm{~ms})$, only the 9 first images, with TE multiples of $22.5 \mathrm{~ms}$, were used. Fig. 3 shows the fit of the mean intensity versus TE, for both liver and spleen regions marked on Fig. 2. As T1 for these tissues are shorter than the TR, a single-exponential curve gives a good fit.
Nowadays, there has been a marked increase in the use of MRI for breast tissue evaluation. Multiple research studies have confirmed the potential of relaxometry for early breast cancer detection, diagnosis, and evaluation of response to therapy [16]. From T2-weighted images, differentiation between adipose and glandular tissue types is clear due to their distinctly different T2 values. For the same reason relaxometry differentiate easily normal from abnormal tissue.

Figure 4 shows a breast MRI T2-weighted image (TE=202.5 ms, TR=2000 ms) from a 54 year old woman. The R2 map was evaluated using the same protocol of acquisition and processing presented previously. Fig. 5 shows the relaxation curve fitted by a single-exponential curve for the three different regions of breast indicated in Fig. 4. 


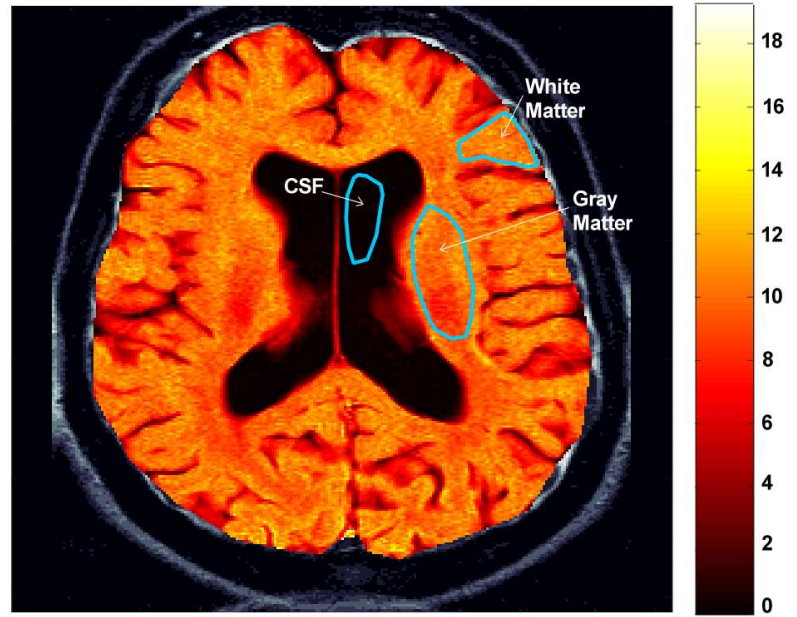

FIG. 6: Map of relaxation rate R2 of an axial slice from a human brain superposed on T2-weighted image. Regions with short T2 are indicated with bright color and long $\mathrm{T} 2$ with black color.

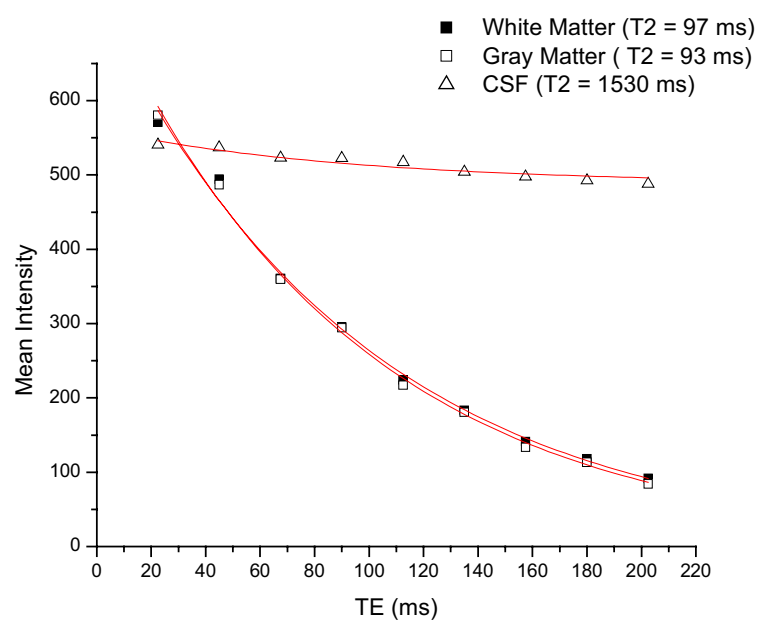

FIG. 7: Single-exponential fit of mean intensity signal versus TE from different brain tissues of an axial slice.

\section{Brain Relaxometry}

T2 relaxometry in human brain has also been successfully used to differentiate normal from abnormal tissues. Increased signal on T2-weighted MRI is a feature to identify cerebral abnormalities. The measurement of $\mathrm{T} 2$ has been shown to be useful in the assessment of hippocampal sclerosis, particularly if there are only subtle changes that may not be evident visually, in the evaluation of some tissue regions as contralateral hippocampus, amygdale, white matter, and thalamus $[1,2,17]$.

Figure 6 shows an R2 map of an axial slice from a 48 year old woman brain, superimposed onto a $\mathrm{T} 2$ weighted image. This map was generated by fitting single-exponentials of corresponding pixels of images obtained using the same multispin sequence and protocol of processing presented in the previous sections.
Due to the large number of subdivisions of brain anatomy, sometimes it is difficult to select a small region to perform relaxometry. Nevertheless, with a whole brain map, small segmented regions are selected and the mean T2 value, or histogram, for each segment can be generated. Fig. 7 shows the single-exponential fit for the three regions selected on Fig. 6. The T2 values in white and gray matter were 97 and $93 \mathrm{~ms}$, respectively. For the CSF T2 was $1,530 \mathrm{~ms}$.

Table 3 shows the relaxometry parameters for liver, spleen, breast and brain tissues, determined using the same procedure used for the simulated study. The signal of offset was estimated getting the signal intensity when the TE was infinite. A fitting was also made for zero offset.

\section{DISCUSSION}

In this study, we observed that when the $\mathrm{T} 2$ is being determined by a single-exponential fit, the offset should be measured and maintained fixed when evaluating variation on relaxometry measurement caused by abnormalities on a specific region. When using a linear fit to the natural logarithm of the signal intensity without first subtracting an offset amplitude, the $\mathrm{T} 2$ of the tissue will be uncertain and the data will not be well fit. The relaxometry parameter will be unable to evaluate small abnormalities in tissue. For example, when only two echoes are used for a relaxometry measurement, it is impossible to predict an offset signal and the T2 value is obtained by linear fitting of the natural logarithm of the signal intensity versus TE.

In fibroglandular and adipose breast tissues the relaxation times ( $\mathrm{T} 2{ }_{1}$ and $\left.\mathrm{T} 22\right)$ obtained from a bi-exponential fit were different. It implies that although the data were visually well fitted by a single-exponential function, the relaxation of signal has different rate in different portions of the curve. So, fitting the data using a bi-exponential function with the amplitude of both exponential terms equal $\left(\mathrm{SO}_{1}=\mathrm{SO}_{2}\right)$ and one offset value fixed for the same study, we are looking at the relaxometry rate of segments that are proton density and $\mathrm{T} 2$ - weighted separately. If the image was acquired using the ideal range for TE adjusted on sequence, the amplitude obtained from this last protocol of fitting should be approximately equal to the amplitude of signal for TE equal to T2 of the tissue.

The R2 map has a contrast equivalent to a T2 weighted image with the advantage of representing quantitative information that is extremely useful for abnormality diagnosis. In summary care must be taken to standardize the whole procedure for proton relaxation measurements in MRI in order to give clinical value to this parameter.

In conclusion, this work emphasizes the principals of the relaxation of the protons in different tissue environments and how handling the data to extract the relaxometry parameters as quantitative diagnostic with low error. In practical, the MR signal comes simultaneously from relaxation of protons present in different environments, each with distinct density and $\mathrm{T} 2$ value, being the signal a sum of all contributions like equation 1. Because of that, a bi-exponential fitting is extremely necessary, except in cases when TR is much longer 
TABLE III: Relaxometry parameter obtained from Single- and Bi-exponential fitting of MRI signal from a spin-echo sequence versus TE on liver, spleen, breast and brain region. The superscript " $F$ " means fixed parameter during the fitting.

\begin{tabular}{|c|c|c|c|c|c|c|c|c|}
\hline \multirow{2}{*}{ Tissue } & \multicolumn{3}{|c|}{ Single-Exponential } & \multicolumn{5}{|c|}{ Bi-Exponential } \\
\hline & $\mathrm{y} 0$ & $\mathrm{~S}_{o}$ & T2 & $\mathrm{y} 0$ & $\mathrm{~S}_{o 1}$ & $\mathrm{~T} 22_{1}$ & $\mathrm{~S}_{o 2}$ & $\mathrm{~T} 2_{2}$ \\
\hline \multirow{2}{*}{ Liver } & 23.7 & 648.4 & 55 & 24.8 & 307.2 & 54.2 & 342.7 & 55.1 \\
\hline & $0^{F}$ & 636.5 & 63.0 & $23^{F}$ & 323.9 & 55.3 & 323.9 & 55.3 \\
\hline \multirow{2}{*}{ Spleen } & 2.8 & 484.5 & 73.9 & 2.8 & 242.8 & 73.9 & 243.8 & 73.9 \\
\hline & $0^{F}$ & 484.5 & 75.3 & $2.8^{F}$ & 242.1 & 73.9 & 242.1 & 73.9 \\
\hline \multirow{2}{*}{ Breast: Fibroglandular } & 23.3 & 1012.7 & 65.9 & 23.3 & 705.6 & 65.9 & 307.0 & 70.0 \\
\hline & $0^{F}$ & 993.7 & 73.5 & $23.3^{F}$ & 512.2 & 64.8 & 512.2 & 72.0 \\
\hline \multirow{2}{*}{ Breast: Adipose } & 165.3 & 1931.0 & 75 & 165.3 & 964.0 & 75.0 & 967.6 & 75.0 \\
\hline & $0^{F}$ & 1970 & 96.7 & $165.3^{F}$ & 965.8 & 75.0 & 965.8 & 75.0 \\
\hline \multirow{2}{*}{ Breast: Lesion } & 38.7 & 1241.9 & 146.3 & 38.7 & 1160.0 & 190 & 273.7 & 32.0 \\
\hline & $0^{F}$ & 1333.7 & 206.3 & $38^{F}$ & 709.0 & 105.3 & 709.0 & 37.0 \\
\hline \multirow[b]{2}{*}{ Brain: CSF } & 40 & 514.6 & 1638 & 42.6 & 256.0 & 1629 & 256 & 1629 \\
\hline & $0^{F}$ & 568.3 & 1767 & $40^{F}$ & 257.0 & 1631.0 & $257.0^{F}$ & 1631 \\
\hline \multirow{2}{*}{ Brain: Gray Mater } & 18.6 & 745.1 & 90.9 & 18.0 & 522.4 & 90.9 & 222.7 & 90.9 \\
\hline & $0^{F}$ & 741.1 & 100.2 & $18^{F}$ & 372.5 & 91.2 & $372.5^{F}$ & 91.2 \\
\hline \multirow{2}{*}{ Brain: White Matter } & 13.4 & 749.6 & 89.6 & 13.4 & 524.8 & 89.6 & 224.8 & 89.6 \\
\hline & $0^{F}$ & 746.5 & 96.1 & $13^{F}$ & 347.7 & 89.8 & 347.7 & 89.8 \\
\hline
\end{tabular}

than TE. Because of the dispersion on MR signal, due to the intrinsic noise of MRI technique, the fitting of the data is critical and a special attention is also necessary during mathematical processing.

\section{Acknowledgments}

The authors are grateful to Prof. D. T. Covas, M. A. Zago, A. C. Santos, J. Elias Jr., V. Muglia, and A. Martinelli for providing access to in vivo data and many discussions, C. A. Brunello, L. Rocha, E. Navas and L. Aziani for technical support, and FAPESP, CNPq and CAPES for partial financial support.
[1] J. S. Duncan, P. Bartlett, and G. J. Barker, Am. J. Neuroradiology 17, 1805 (1996).

[2] G. D. Jackson, A. Connelly, J. S. Duncan, R. A. Grunewald, D. G. Gadian, Neurology 43, 1793 (1993).

[3] J. Vymazal, M. Hajek, N. Patronas, J. N. Giedd, J. W. M. Bulte, C. Baumgarner, V. Tran, and R. A. Brooks, J. Mag. Res. Imaging 5, 554 (1995).

[4] S. Yuen, K. Yamada, Y. Kinosada, S. Matsushima, Y. Nakano, M. Goto, and T. Nishimura, J. Mag. Res. Imaging 20, 56 (2004).

[5] O. Baffa, A. Tannus, M. A, Zago, M. S. Figueiredo, and H. C. Panepucci, Bull Magn. Reson 8, 69 (1986).

[6] A. A. O. Carneiro, J. P. Fernandes, D. B. de Araujo, J. Elias Jr., A. L. C. Martinelli, D. T. Covas, M. A. Zago, I. L. Angulo, T. G. St Pierre, and O. Baffa, Mag. Res. Med. 54, 122 (2005).

[7] P. R. Clark and T.G. St Pierre, Mag. Res. Imaging 18, 431 (2000).

[8] J. P. Kaltwasser, R. Gottschalk, K. P. Schalk, and W. Hartl, Brit. J. Haematology 74, 360 (1990).

[9] T. G. St Pierre, P. R. Clark, and W. Chua-anusorn, NMR in Biomedicine 17, 446 (2004).
[10] Y. De Deene, R. Van de Walle, E. Achten, and C De Wagter, Sig. Processing 70, 85 (1998).

[11] R. Engelhardt, J. H. Langkowski, R. Fischer, P. Nielsen, H. Kooijman, H. C. Heinrich, and E. Bucheler, Mag. Res. Imaging 12, 999 (1994).

[12] D. Gensanne, G. Josse, J. M. Lagarde, D. Vincensini, Phys. Med. Biol. 50, 3755 (2005).

[13] E. M. Bellon, E. M. Haacke, P. E. Coleman, D. C. Sacco, D. A. Steiger, and R. E. Gangarosa, Am. J. Roentgenology 147, 1271 (1986).

[14] P. R. Clark, W. Chua-anusorn, and T. G. St Pierre, Comput. Med. Imag. Graph. 28, 69 (2004).

[15] R. E. Hendrick, J. B. Kneeland, and D. D. Stark, Mag. Res. Imaging 5, 117 (1987).

[16] C. D. Lehman and M. D. Schnall, Breast Cancer Res. 7, 215 (2005).

[17] R. C. Scott, F. M. C. Besag, and B. G. Neville, Brit. Med. J. 322, 107 (2001) 